\title{
Cyclophilins in Ischemic Heart Disease: Differences Between Acute and Chronic Coronary Artery Disease Patients
}

\author{
Jeremias Bayon $^{\mathrm{a}}$, Amparo Alfonso ${ }^{\mathrm{b}}$, Sandra Gegunde ${ }^{\mathrm{b}}$, Eva Alonso ${ }^{\mathrm{b}, \mathrm{c}}$, Rebeca Alvarino ${ }^{\mathrm{b}}$, \\ Melisa Santas-Alvarez ${ }^{\mathrm{a}}$, Ana Testa-Fernandez ${ }^{\mathrm{a}}$, Ramon Rios-Vazquez ${ }^{\mathrm{a}}$, \\ Luis Botana ${ }^{\mathrm{b}}$, Carlos Gonzalez-Juanatey ${ }^{\mathrm{a}, \mathrm{d}}$
}

\begin{abstract}
Background: Cyclophilins (Cyps) are a family of peptidyl-prolyl cis/trans isomerases consistently involved in cardiovascular diseases through the inflammation pathway. This study aims to investigate the serum levels of Cyps (CypA, CypB, CypC and CypD) in patients with coronary artery disease (CAD) and the correlation with clinical characteristics and inflammation parameters.

Methods: We developed an observational prospective study with a total of 125 subjects: 40 patients with acute CAD, 40 patients with chronic $\mathrm{CAD}$ and 45 control volunteers, in whom serum levels of Cyps (CypA, CypB, CypC and CypD), interleukins and metalloproteinases were measured.

Results: CypA levels increased significantly in CAD patients compared with control subjects, but no differences were noted between acute CAD $(7.80 \pm 1.30 \mathrm{ng} / \mathrm{mL})$ and chronic CAD $(5.52$ $\pm 0.76 \mathrm{ng} / \mathrm{mL})$ patients $(\mathrm{P}=0.13)$. No differences in CypB and CypD levels were showed between CAD patients and controls and between acute $\mathrm{CAD}$ and chronic CAD patients. In relation with $\mathrm{CypC}$, the levels in CAD patients were significantly higher compared to controls $(32.42 \pm 3.71 \mathrm{pg} / \mathrm{mL}$ vs. $9.38 \pm 1.51 \mathrm{pg} / \mathrm{mL}, \mathrm{P}<$ $0.001)$, but no differences between acute and chronic CAD groups were obtained $(\mathrm{P}=0.62)$. We analyzed the CypC $>17.5 \mathrm{pg} / \mathrm{mL}$ cut-off point, and it was significantly associated with older age, hypertension, dyslipidemia and more extensive CAD in acute and chronic CAD groups.
\end{abstract}

Manuscript submitted June 12, 2020, accepted July 2, 2020

Published online August 1, 2020

${ }^{a}$ Cardiology Department, Hospital Universitario Lucus Augusti, c/Ulises Romero ${ }^{\circ}$ 1, 27003 Lugo, Spain

bPharmacology Department, Facultad de Veterinaria, Universidad de Santiago de Compostela, Avenida Carballo Calero s/n, 27002 Lugo, Spain

${ }^{c}$ Fundacion Instituto de Investigacion Sanitario Santiago de Compostela (FIDIS), Hospital Universitario Lucus Augusti, 27003 Lugo, Spain

${ }^{\mathrm{d}}$ Corresponding Author: Carlos Gonzalez-Juanatey, Cardiology Department, Hospital Universitario Lucus Augusti, c/Ulises Romero n 127003 Lugo, Spain. Email: carlos.gonzalez.juanatey@sergas.es

doi: https://doi.org/10.14740/cr1120
Conclusions: CypA and CypC levels are increased in CAD patients. High CypC serum levels could be a novel biomarker in CAD patients correlating with a more severe disease.

Keywords: Cyclophilins; Inflammation; Ischemic heart disease; Cardiovascular risk factors

\section{Introduction}

Inflammation plays a pivotal role in atherogenic process, being the cornerstone in coronary artery disease (CAD). Proinflammatory cytokines and immune factors are involved in the pathogenesis and progression of atherosclerosis, myocardial ischemia/reperfusion injury and heart failure [1]. Immunophilins are a family of peptidyl-prolyl cis/trans isomerases (PPIases) consistently involved in cardiovascular diseases [2]. PPIases include three protein subfamilies: cyclophilins (Cyps), FK506-binding proteins (FKBP) and parvulins. Cyps are some of the most conserved proteins present in eukaryotes and prokaryotes, and they have been implicated in diverse cellular processes and responses to multiple biotic and abiotic stresses [3]. Some of these proteins, such as CypA, CypB, CypD or FKBP51, have been previously associated with CAD [4-6].

The role of CypA in cardiovascular diseases as a biomarker for CAD has been previously reported [7-9], but less data are available about other Cyps. There is an important role for extracellular CypA in the pathogenesis of several diseases through receptor (cluster of differentiation 147 (CD147) or other) mediated autocrine and paracrine signaling pathways [10]. CypA reduced reactive oxygen species production and suppressed $\mathrm{O}_{2}$ production dependent on reduced nicotinamide adenine dinucleotide phosphate (NADPH) oxidase, which could be a potential target for ischemia-reperfusion injury therapy [11]. Also, CypA has also been involved in arrhythmogenic cardiomyopathy remodeling, characterized by the progressive substitution of functional myocardium with non-contractile fibro-fatty tissue contributing to ventricular arrhythmias and sudden cardiac death [12]. Extracellular Cyps levels are considered as an inflammatory response to injury in many inflammatory processes [13]. In addition, Cyps are related to redox homeostasis, and redox-mediated signal- 
ing has an important role in inflammation, atherosclerosis and CAD $[14,15]$. In another way, inhibiting CypD to inhibit mitochondria permeability transition pore opening protects against ischemia-reperfusion injury, making modulation of CypD activity a potentially important therapeutic goal [16].

CypC has a key role in inflammation related to macrophages activation and has been recently proposed by our group as a novel biomarker in acute CAD patients [17]. In addition, a significant correlation between traditional cardiovascular risk factors and CypC levels were observed in this group of patients [17]. Nevertheless, multiple biomarkers in CAD (troponins, N-terminal pro-brain natriuretic peptide and others) play a key role for diagnosing and prognostication in the acute phase of the events, losing prominence in chronic CAD. We suggest that Cyps could be a marker of coronary disease beyond the acute coronary event, so we studied its levels and the relation with other CAD biomarkers in the acute and chronic phase of CAD.

The aim of the present study was to explore the relationship between Cyps and ischemic heart disease in acute and chronic CAD patients.

\section{Materials and Methods}

\section{Population study}

This is an observational prospective study where 125 subjects (40 patients with acute CAD, 40 patients with chronic CAD and 45 control volunteers) were consecutively enrolled. Acute CAD was defined as unstable angina, non-ST segment myocardial infarction or ST-segment myocardial infarction according current European Society of Cardiology (ESC) practical clinical guidelines $[18,19]$. Chronic CAD was defined as an ischemic heart disease clinically stable without a rise of myocardial biomarkers, as the ESC guidelines defined [20]. Patients were recruited at the Cath-Lab Unit after confirm that they had CAD. All acute CAD patients were admitted to hospital due to an acute coronary syndrome, and all chronic CAD patients were outpatients who came to make a coronary angiography due to clinical indication.

Peripheral blood samples were obtained from all subjects. The blood was centrifuged $\left(3,000 \mathrm{rpm}, 10 \mathrm{~min}\right.$ at $\left.4{ }^{\circ} \mathrm{C}\right)$ and supernatants were collected and stored at $-80{ }^{\circ} \mathrm{C}$ until Cyps analysis. After thawed at room temperature, these supernatants were used to measure levels of Cyps (CypA, CypB, CypC and CypD) by enzyme-linked immunosorbent assay (ELISA) kits. Absorbance measurements were done in a microplate reader at 450 and $540 \mathrm{~nm}$ for CypA, СурB and CypC, and at 450 $\mathrm{nm}$ in the case of CypD. Samples were always run by duplicate. The range of determination was $3.12-200 \mathrm{ng} / \mathrm{mL}$ for СypA; 31.25 - 2,000 pg/mL for CypB; 23.5 - 1,500 pg/mL for CypC and 62.5 - 4,000 pg/mL for CypD. Serum levels below the lower limit of quantification were undetectable and therefore were considered as $0 \mathrm{pg} / \mathrm{mL}$ for statistical analysis. The intra and inter-assay coefficients of variation of the ELISA kits were $<10 \%$. No cross reactivity was observed between Cyp antibodies. Human cyclophilin A ELISA kit (CSB-E09920H), human cyclophilin B ELISA kit (CSB-E11218H) and human cyclophilin C ELISA kit (CSB-EL018473HU) were obtained by Cusabio. Human Cyclophilin D ELISA kit (E-EL-H1936) was from Elabscience.

Levels of serum interferon $\gamma$ (IFN- $\gamma$ ), interleukin (IL)- $1 \beta$, IL-2, IL-6, IL-8, tumor necrosis factor $\alpha$ (TNF- $\alpha)$ and metalloproteinase (MMP)-2 and MMP-9 were determined in serum samples using a magnetic bead-based multiplex immunoassay (Milliplex ${ }^{\circledR}$ Map Kit) according to manufacturer's instructions. Human high sensitivity $\mathrm{T}$ cell magnetic bead panel Milliplex ${ }^{\circledR}$ map kit (\#HSTCMAG-28SK; \#HSTCMAG28SPMX13; \#HSTCMAG28SOMX21; \#HSTCMAG28PMX13BK and \#HSTCMAG28PMX21BK) and human MPP magnetic bead panel 2 Milliplex ${ }^{\circledR}$ map kit (\#HMMP2MAG-55K) were purchased from Merck (Madrid, Spain).

Clinical characteristics were obtained for all the patients and control volunteers from their medical history.

The institutional and regional ethical board approved the study (reference: 2016/508, approved date: December 19, 2016, according to the principles outlined in the Declaration of Helsinki). Participants in the present study were informed and a voluntarily written informed consent was obtained from all of them.

\section{Statistics}

SPSS24 for windows was used for the statistical analysis. Categorical variables were presented as percentages and continuous variables were presented as means \pm standard error of the mean (SEM). Kolmogorov-Smirnov test (with Lilliefors correction) was first performed as a normality test.

Comparisons between three groups (controls, acute CAD and chronic $\mathrm{CAD}$ ) were made; comparisons between the controls and CAD patients were also made.

Statistical significance in qualitative variables was calculated with Chi-square test. Those continuous variables with normal distribution were compared between two groups using a Student's $t$-test (including Levene's test to assess the equality of variance), otherwise the non-parametric Mann-Whitney test was used. Differences between three groups were calculated using analysis of variance (ANOVA) test.

Receiver-operating-characteristic curves (ROC) were constructed to assess the sensitivity and specificity of Cyps and were used to obtain optimal cut-off points for Cyps in relation to $\mathrm{CAD}$.

Three models of parsimonious logistic regression were constructed to evaluate if relationship between Cyps and CAD was independent of other factors related to CAD.

\section{Results}

We studied 125 subjects (mean age $57.8 \pm 14.5$ years; $72.8 \%$ male): 40 acute CAD patients (35 men and five women), 40 chronic CAD patients (33 men and seven women) and 45 control volunteers without CAD (23 men and 22 women). The same measurements were done in both populations (CAD patients and controls) and results were compared.

Baseline characteristics and biochemical data were col- 


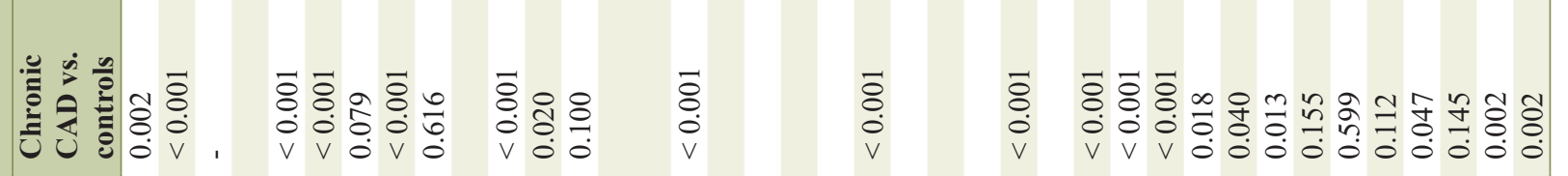

站:

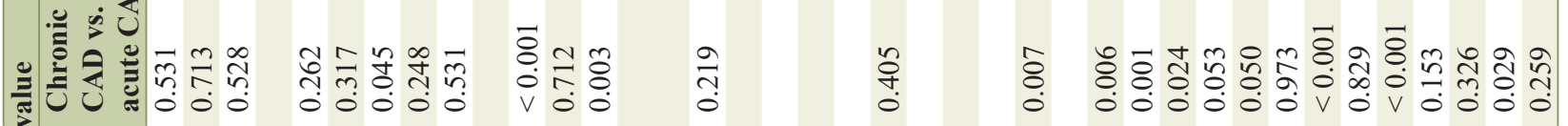

a

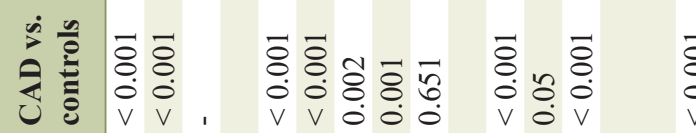

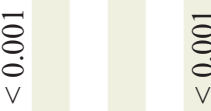

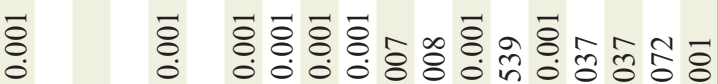

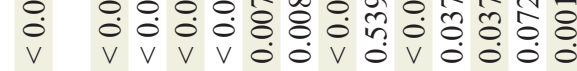

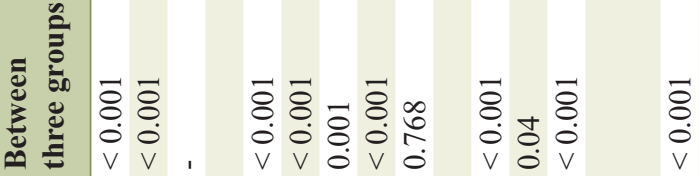

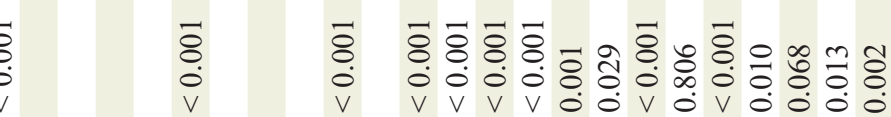

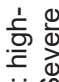

$\dot{0}$

$\frac{\check{C}}{\mathscr{\Phi}}$

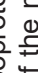

$\stackrel{\circ}{=}$

ते 흔

焉

家

(

कृ

응응

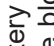

紊官

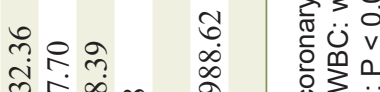

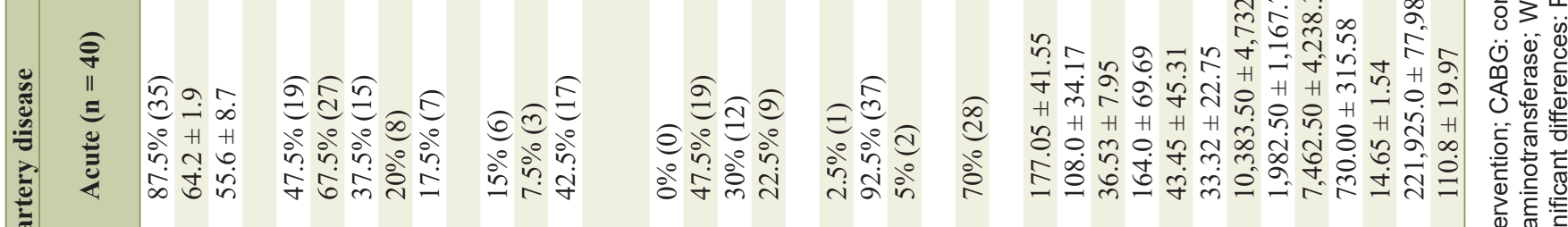
उ $\frac{\omega}{4}$ ठำ

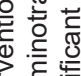
ㄴ. نั:

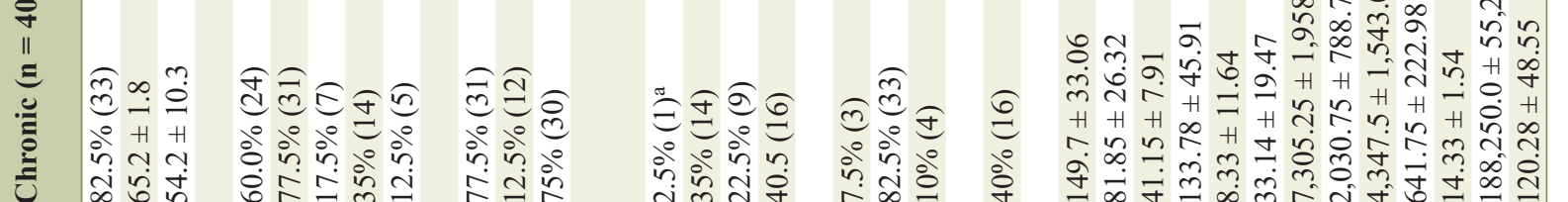
需

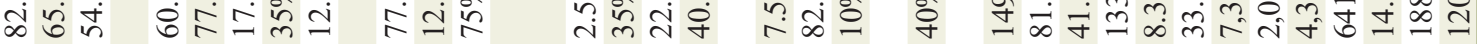


lected in the three groups and showed in Table 1.

Patients with CAD compared with controls had more traditional cardiovascular risk factors. Triglycerides (TG), high sensitivity C-reactive protein (hsCRP) and alanine transaminase (ALT) were significantly increased in CAD patients while cholesterol values were significantly decreased, according to statin treatment in this very high-risk population. HsCRP levels were significantly increased in acute CAD vs. chronic CAD patients $(43.45 \pm 45.31 \mathrm{mg} / \mathrm{dL}$ vs. $8.33 \pm 11.64 \mathrm{mg} / \mathrm{dL} ; \mathrm{P}=0.05)$, as well as between acute and chronic CAD and control group respectively $(43.45 \pm 45.31 \mathrm{mg} / \mathrm{dL}$ and $8.33 \pm 11.64 \mathrm{mg} / \mathrm{dL}$ vs. $0.49 \pm 0.5 \mathrm{mg} / \mathrm{dL} ; \mathrm{P}=0.007)$, with no significant correlation between Cyps and hsCRP serum levels among the groups. In the case of blood cell count, white blood cell, neutrophils, monocytes and hemoglobin were significantly increased in CAD patients as well as glucose levels, while lymphocytes and platelets were not modified. No differences between acute CAD and chronic CAD were observed in the number of coronary vessels involved in CAD and in left ventricular ejection fraction. Otherwise, we observed a significant difference in the complete coronary artery revascularization $(70 \%$ acute CAD vs. $40 \%$ chronic CAD; $\mathrm{P}=0.007)$. Serum levels of CypA, CypB, CypC and CypD are showed in Table 2. Considering CAD patients as a group, CypA levels were significantly increased in CAD patients $(6.65 \pm 0.75 \mathrm{ng} / \mathrm{mL}$ compared with control subjects: $2.53 \pm 0.53 \mathrm{ng} / \mathrm{mL} ; \mathrm{P}<0.001)$. No significant differences were noted between acute $\mathrm{CAD}$ group and chronic CAD group (7.80 $\pm 1.30 \mathrm{ng} / \mathrm{mL}$ vs. $5.52 \pm 0.76 \mathrm{ng} / \mathrm{mL} ; \mathrm{P}=0.134)$. The CypC levels in CAD patients were significantly higher compared with control subjects $(32.42 \pm 3.71 \mathrm{pg} / \mathrm{mL}$ vs. $9.38 \pm 1.51 \mathrm{pg} / \mathrm{mL}$; $\mathrm{P}<0.001)$, but there were no differences between acute and chronic CAD groups $(34.28 \pm 5.77 \mathrm{pg} / \mathrm{mL}$ vs. $30.56 \pm 4.73 \mathrm{pg} /$ $\mathrm{mL} ; \mathrm{P}=0.620)$. Also, there were no differences between CAD patients and control groups in $\mathrm{CypB}$ and CypD levels. No significant differences in the plasma levels of IL-2, IL-6, TNF- $\alpha$, and MMP-9 were observed when CAD patients and controls were compared. IL- $1 \beta$ shows high levels in CAD patients (with no differences between groups: $3.94 \pm 0.42 \mathrm{pg} / \mathrm{mL}$ in chronic CAD vs. $3.73 \pm 0.37 \mathrm{pg} / \mathrm{mL}$ in acute $\mathrm{CAD} ; \mathrm{P}=0.69)$ than controls $(2.71 \pm 0.17 \mathrm{pg} / \mathrm{mL} ; \mathrm{P}<0.001)$. IL-6 levels were significantly increased in acute CAD vs. chronic CAD patients $(8.08$ $\pm 1.59 \mathrm{pg} / \mathrm{mL}$ vs. $3.95 \pm 0.59 \mathrm{pg} / \mathrm{mL} ; \mathrm{P}=0.019)$ as well as between acute CAD and control group $(8.08 \pm 1.59 \mathrm{pg} / \mathrm{mL}$ vs. $4.04 \pm 0.33 \mathrm{pg} / \mathrm{mL} ; \mathrm{P}=0.015)$. In the case of IL- 8 a significant decrease in CAD patients' serum levels were observed (6.86 \pm $0.88 \mathrm{pg} / \mathrm{mL}$ for CAD patients vs. $8.17 \pm 0.65 \mathrm{pg} / \mathrm{mL}$ for control subjects; $\mathrm{P}=0.037)$. The same happens when IFN- $\gamma$ levels were measured, since a decrease in CAD patients $(27.5 \pm 2.4 \mathrm{pg} / \mathrm{mL}$ in chronic $\mathrm{CAD}$ and $27.4 \pm 2.7 \mathrm{pg} / \mathrm{mL}$ in acute $\mathrm{CAD}$ ) vs. controls $(34.3 \pm 2.8 \mathrm{pg} / \mathrm{mL} ; \mathrm{P}=0.03)$ was observed. MMP-2 levels were significantly decreased, from $120,317 \pm 5,068 \mathrm{ng} / \mathrm{mL}$ in control subjects to $100,152 \pm 3,820 \mathrm{ng} / \mathrm{mL}$ in CAD patients ( $\mathrm{P}$ $=0.003)$, with no differences between CAD groups $(95,434 \pm$ $5,691 \mathrm{ng} / \mathrm{mL}$ in acute CAD group vs. $106,524 \pm 5,010 \mathrm{ng} / \mathrm{mL}$ in chronic CAD group; $\mathrm{P}=0.148$ ).

Cyps levels were linked with traditional cardiovascular risk factors. CypA was significantly linked to hypertension, dyslipidemia, smoking, being male and being over 50 years old. CypB was linked to hypertension and being female. How-

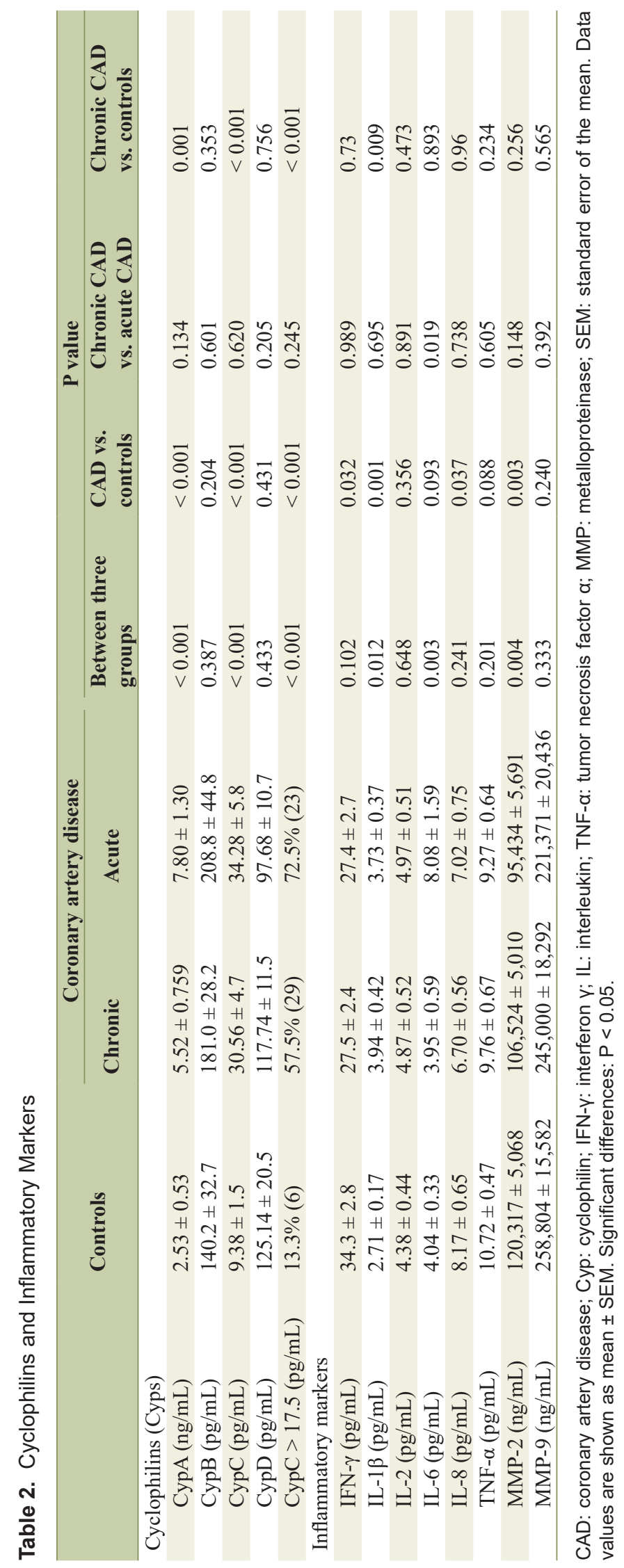



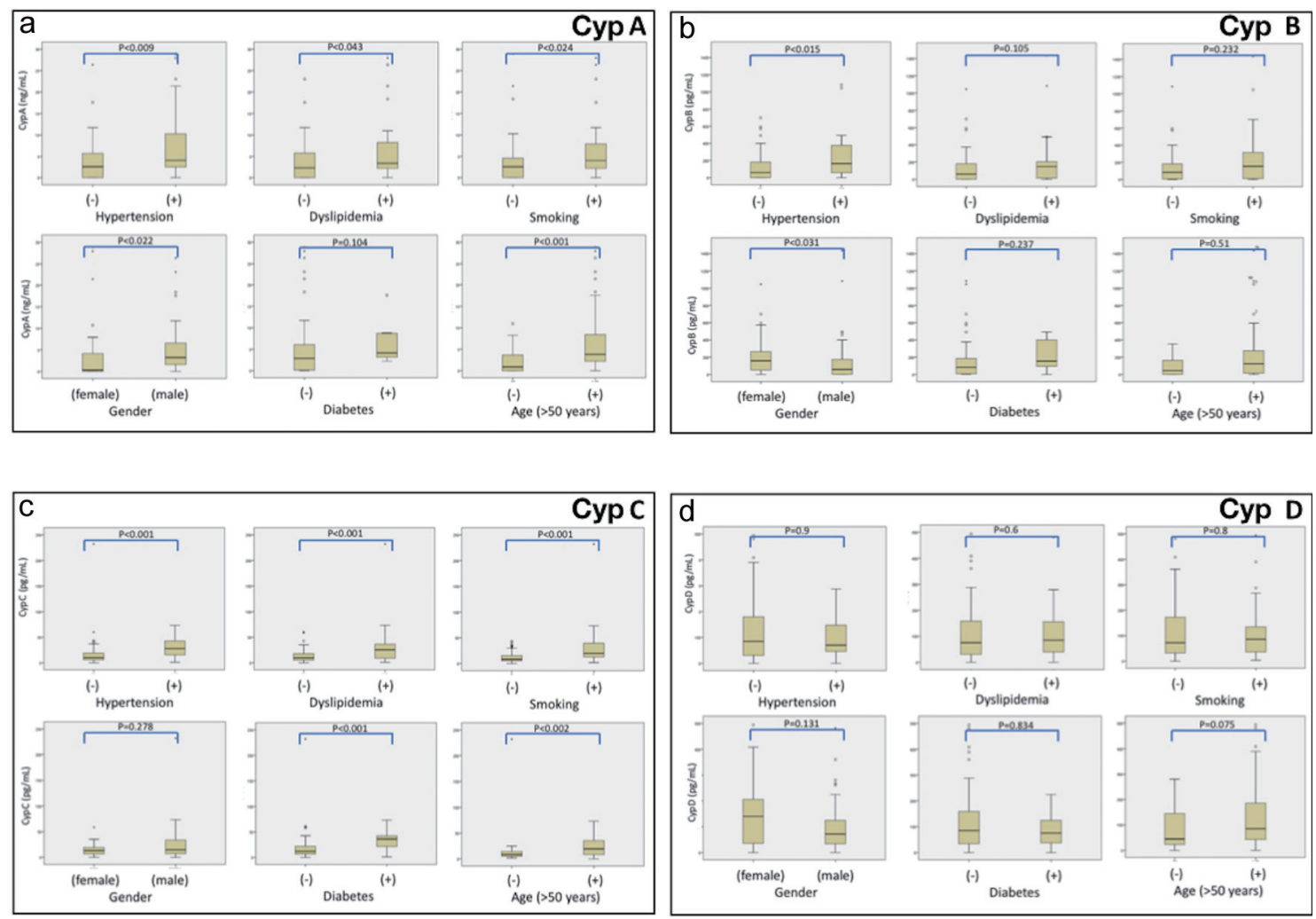

Figure 1. Serum Crps levels ((a) CypA, (b) CrpB, (c) CypC, (d) CypD) in patients with CAD and the relationship with traditional cardiovascular risk factors. Frequency plots: data represented as Cyp levels in x-axis, versus traditional cardiovascular risk factors (hypertension, dyslipidemia, smoking, gender, diabetes and age > 50 years) in y-axis. CAD: coronary artery disease; Crps: cyclophilins.

ever, CypC was significantly linked to hypertension, dyslipidemia, smoking, diabetes and being over 50 , but not being male. Finally, CypD was not linked to the traditional cardiovascular risk factors involved in our study (Fig. 1).

ROC curves were calculated on the basis of Cyps levels, using CAD presence or absence as state variable. Higher sensitivity and selectivity values were obtained with CypC. The area under the ROC curve (AUC) (c-statistic) calculated was 0.85 , showing a significant value $(\mathrm{P}<0.001)$ of CypC as predictor of CAD. For CypA and CypB, AUC was also significant, 0.74 and 0.65 respectively, with less predictive power, while CypD values are not useful to predict CAD (AUC: $0.56)$.

In our study, the optimal cut-off point as CAD marker was CypC $>17.5 \mathrm{pg} / \mathrm{mL}$, and we recently proposed this value as a novel biomarker of CAD in acute CAD patients [17]. Using this CypC cut-off point, we observed that it was present in $72.5 \%$ of acute CAD patients, and $57.5 \%$ in chronic CAD patients but only in $11.1 \%$ of controls subjects. We also observed that this cut-off point is significantly linked to older age, hypertension, dyslipidemia and presence of CAD and more extensive CAD (Table 3).

The cut-off point of Cyp $>17.5 \mathrm{pg} / \mathrm{mL}$ for the diagnosis of CAD had a sensibility of $63.7 \%$ and a specificity of $88.9 \%$ with a positive predictive value of $91.1 \%$ and a negative predictive value of $58.0 \%$. We made three models of logistic regression, with variables that had shown a significant link with the presence of CAD in univariable analysis, in order to explore if this cut-off point was independent from the presence of CAD. This multivariable analysis confirms the strong association of CypC $>17.5 \mathrm{pg} / \mathrm{mL}$ cut-off point with CAD (odds ratio (OR): 56.4; $\mathrm{P}=0.019$ ) even when we adjusted with potential confounding factors. Also, total cholesterol, low-density lipoprotein cholesterol (LDL-C), age and male gender are significantly linked to CAD (Table 4).

\section{Discussion}

Inflammation has a key role in cardiovascular diseases, and Cyps are implicated in this mechanism [7, 10]. CypA plasma levels have been confirmed as biomarkers for CAD, even with prognosis impact in long-term follow-up [9]. On the other hand, the rest of Cyps are not well known yet, and their relationship with $\mathrm{CAD}$ is a fact worth studying further. In our study, we described the serum levels of four Cyps and eight inflammation markers in patients with CAD (acute CAD and chronic $\mathrm{CAD}$ ) and controls, with the objective of looking for association with $\mathrm{CAD}$

CypA is released to the extracellular media in response to inflammation. CypA is involved in multiple processes such as endothelial dysfunction, modulation of smooth muscle cells, 
Table 3. Univariate Analysis of CypC $\geq 17.5 \mathrm{pg} / \mathrm{mL}$ : Clinical Characteristics

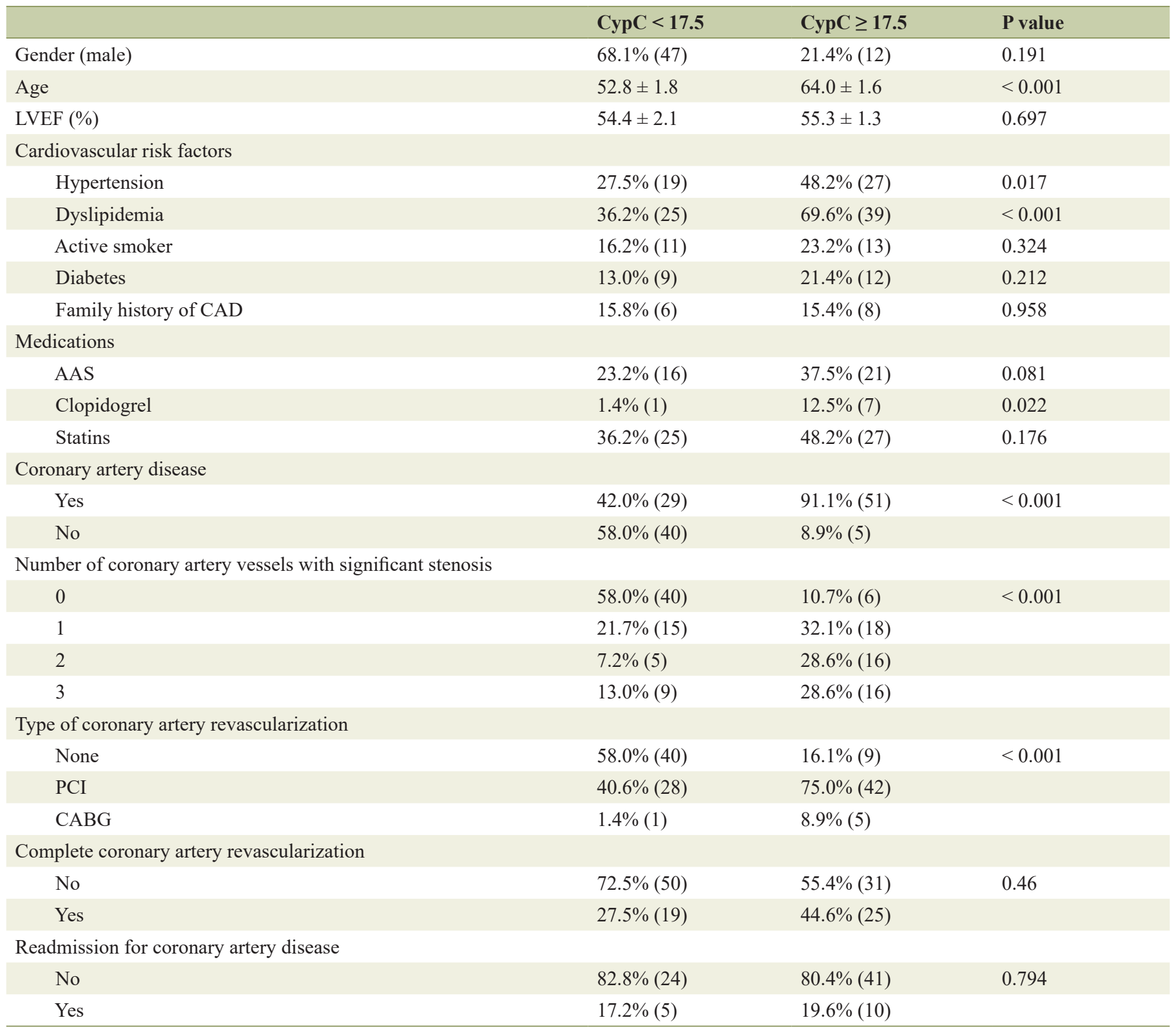

LVEF: left ventricular ejection fraction; AAS: acetylsalicylic acid; PCl: percutaneous coronary intervention; CABG: coronary artery bypass graft. Significant differences: $\mathrm{P}<0.05$.

fibroblast and cardiac hypertrophy [2, 5]. Even more, CypA levels can be used to predict all-cause death, rehospitalization, and coronary revascularization in patients with CAD [9]. Also, in patients with acute myocardial infarction, platelet-bound CypA was significantly decreased compared with patients with stable CAD [21]. In our study, CypA levels were significantly increased in CAD patients, in the same line of previous studies $[9,14]$.

CypB has a crucial role against ROS and pro-inflammatory status, and their levels were associated with metabolic syndrome, vascular function and heart failure [2,6]. Even though, in our study, we do not find any difference between CAD pa- tients and controls in CypB levels.

Cyp $\mathrm{C}$ has a key role in inflammation and has been recently published by our group as a novel biomarker in acute CAD patients [17]. The main finding of this study is that in our population, CypC is strongly correlated with the presence of chronic and acute CAD and with a cut-off point of CypC $>17.5 \mathrm{pg} / \mathrm{mL}$; we can classify patients of CAD with high sensibility of $63.7 \%$ and specificity of $88.9 \%$. In our study, the cut-off point of $>17.5 \mathrm{pg} / \mathrm{mL}$ was associated with more extensive CAD (two or three coronary vessels with significant stenoses in $56 \%$ of cases vs. $20.2 \%$ in patients with lower levels) and related to the need for coronary revascular- 
Table 4. Multivariate Analysis: CypC $>17.5 \mathrm{pg} / \mathrm{mL}$ and Related Factors With Presence of CAD

\begin{tabular}{|c|c|c|c|}
\hline & OR & OR CI 95\% & P value \\
\hline \multicolumn{4}{|c|}{ Model 1: CypC $>17.5$ and clinical variables } \\
\hline СурС $>17.5$ & 56.808 & $4.85-665$ & 0.001 \\
\hline Gender (male) & 45.4 & $3.13-498$ & 0.005 \\
\hline Hypertension & 13.044 & $1.25-135$ & 0.032 \\
\hline \multicolumn{4}{|c|}{ Model 2: CypC $>17.5$ and analytical variables } \\
\hline СурС $>17.5$ & 22.6 & $3.77-136$ & 0.001 \\
\hline Total cholesterol & 0.830 & $0.751-0.918$ & 0.001 \\
\hline LDL & 1.170 & $1.05-1.30$ & 0.003 \\
\hline Total cholesterol & 0.963 & $0.938-0.989$ & 0.005 \\
\hline Gender (male) & 40.7 & $4.32-383$ & 0.001 \\
\hline Age & 1.215 & $1.095-1.348$ & $<0.001$ \\
\hline
\end{tabular}

CypC: cyclophilin C; LDL: low-density lipoprotein; TG: triglycerides; OR: odds ratio; OR CI 95\%: odds ration confidence interval 95\%. Significant differences: $P<0.05$.

ization procedures ( $86.9 \%$ vs. $42 \%$, respectively). Therefore, in relation to patients with higher CypC levels, it is a promising and significant marker of complex and diffuse CAD. In addition, CypC levels were correlated with age and higher prevalence of hypertension and dyslipidemia. Moreover, it is the first study showing a significant link between CypC levels with chronic CAD, adding additional information to the classic CAD biomarkers, which only increases in the acute scenario.

CypD has been shown to be also associated with inflammation, atherosclerosis and diabetes. The inhibition of CypD is postulated as a cardio-protection target in ischemia-reperfusion myocardial injury [22]. In our study, no differences in CypD levels were observed between CAD groups.

The pro-inflammatory cytokines play a pivotal role in atherosclerosis. IL-1 $\beta$ is a cytokine that is central to the inflammatory response and that drives the IL-6 signaling pathway. In our study, CAD patients had significantly higher IL-1 $\beta$ serum levels than controls. The CANTOS trial (Canakinumab Antiinflammatory Thrombosis Outcome Study) provided the first evidence that targeting inflammation in humans with ischemic heart disease could improve clinical outcomes. Treatment with the anti-IL-1 $\beta$ antibody canakinumab significantly reduced recurrent cardiovascular events in individuals with stable CAD well treated with standard-of-care measures [23]. In addition, in a recent study, High levels of IL-1 $\beta$ were more often present in patients with acute coronary syndrome vs. controls $(55 \%$ vs. $40 \%$ vs. $34 \%, \mathrm{P}<0.001)$, and were predictive for all-cause mortality (adjusted hazard ratio (HR): 1.45; 95\% CI: 1.16 $1.82, \mathrm{P}<0.001)$ [24].
IL-6 plays a central role in inflammation and tissue injury, and long-term IL-6 plasma levels are linked with CAD risk as strongly as some other major established risk factors [25]. In our study, acute CAD patients had significantly higher IL-6 serum levels than chronic CAD patients. It was recently published that IL-6 was higher in patients with functionally relevant CAD as compared to those without $(1.56 \mathrm{pg} / \mathrm{mL}$ vs. $1.30 \mathrm{pg} / \mathrm{mL}, \mathrm{P}<0.001$ ) [26]. On the other hand, IL-8 is the other cytokine that is involved in the ischemic heart disease and in the post-ischemic tissue repair processes and thus is considered to exert beneficial effects on cardiac function [27]. In this study, patients with CAD had significantly lower levels of IL-8 than controls. Finally, we identify lower levels of IFN- $\gamma$ in CAD patients vs. controls. It is known that despite its strong proinflammatory effects, IFN- $\gamma$ also exhibits anti-inflammatory characteristics and, accordingly, is able to manipulate the secretion of both pro- as well as anti-inflammatory signaling molecules.

Taking into account all these observations, it is possible that serum levels of CypC $>17.5 \mathrm{pg} / \mathrm{mL}$ could apply as a possible CAD biomarker in the future.

\section{Limitations}

In this preliminary study, the number of patients studied is small; even so the significance of the relationship between $\mathrm{CypC}$ and the presence of CAD is very relevant. Another limitation is the difference in traditional cardiovascular risk factors between the healthy volunteer group and the CAD group. In 
this regard, we made a multivariate analysis including these variables to confirm the independent relationship between CypC and CAD.

\section{Conclusions}

CypA and CypC levels are increased in CAD patients. No differences in Cyps serum levels between acute and chronic CAD patients were found. High CypC serum levels could be a novel biomarker in CAD patients correlating with a more severe disease.

\section{Acknowledgments}

We appreciated the support of Dr. Emilio Paredes for statistical analysis.

\section{Financial Disclosure}

This study has received funding from the following FEDER co-funded grants: Conselleria de Cultura, Educacion e Ordenacion Universitaria Xunta de Galicia, 2017 GRC GI-1682 (ED431C 2017/01); Ministerio de Ciencia e Innovacion ISCIII/PI16/01816, ISCIII/PI16/01830, ISCIII/PI19/00879 and ISCIII/PI19/001248; and from European Union POCTEP 0161-Nanoeaters-1-E-1, Interreg AlertoxNet EAPA-317-2016, Interreg Agritox EAPA-998-2018 and H2020 778069- EMERTOX. Sandra Gegunde was supported by a fellowship from FIDIS, Spain.

\section{Conflict of Interest}

The authors declare that they have no conflict of interest.

\section{Informed Consent}

All subjects provided written informed consent.

\section{Author Contributions}

JB, AA and CGJ has designed and performed the study. JB, $\mathrm{AA}, \mathrm{EA}$ and LB have drafted the manuscript and did critical editing. JB, AA, SG, RA, MSA, ATF and RRV have assisted and supported in sample collection and subsequent analysis with statistics. JB, AA, LB and CGJ have carefully supervised this manuscript preparation and writing.

\section{Data Availability}

The authors declare that data supporting the findings of this study are available within the article.

\section{References}

1. Goikuria H, Vandenbroeck K, Alloza I. Inflammation in human carotid atheroma plaques. Cytokine Growth Factor Rev. 2018;39:62-70.

2. Perrucci GL, Gowran A, Zanobini M, Capogrossi MC, Pompilio G, Nigro P. Peptidyl-prolyl isomerases: a full cast of critical actors in cardiovascular diseases. Cardiovasc Res. 2015;106(3):353-364.

3. Singh K, Winter M, Zouhar M, Rysanek P. Cyclophilins: less studied proteins with critical roles in pathogenesis. Phytopathology. 2018;108(1):6-14.

4. Ramachandran S, Venugopal A, Kutty VR, A V, G D, Chitrasree V, Mullassari A, et al. Plasma level of cyclophilin $\mathrm{A}$ is increased in patients with type 2 diabetes mellitus and suggests presence of vascular disease. Cardiovasc Diabetol. 2014;13:38.

5. McClements L, Annett S, Yakkundi A, Robson T. The role of peptidyl prolyl isomerases in aging and vascular diseases. Curr Mol Pharmacol. 2015;9(2):165-179.

6. Zhang H, Fan Q, Xie H, Lu L, Tao R, Wang F, Xi R, et al. Elevated serum cyclophilin B levels are associated with the prevalence and severity of metabolic syndrome. Front Endocrinol (Lausanne). 2017;8:360.

7. von Ungern-Sternberg SNI, Zernecke A, Seizer P. Extracellular matrix metalloproteinase inducer EMMPRIN (CD147) in cardiovascular disease. Int J Mol Sci. 2018;19(2).

8. Satoh K. Cyclophilin A in cardiovascular homeostasis and diseases. Tohoku J Exp Med. 2015;235(1):1-15.

9. Ohtsuki T, Satoh K, Omura J, Kikuchi N, Satoh T, Kurosawa R, Nogi M, et al. Prognostic impacts of plasma levels of cyclophilin A in patients with coronary artery disease. Arterioscler Thromb Vasc Biol. 2017;37(4):685693.

10. Xue C, Sowden MP, Berk BC. Extracellular and intracellular cyclophilin A, native and post-translationally modified, show diverse and specific pathological roles in diseases. Arterioscler Thromb Vasc Biol. 2018;38(5):986993.

11. Cheng F, Yuan W, Cao M, Chen R, Wu X, Yan J. Cyclophilin A Protects Cardiomyocytes against Hypoxia/ Reoxygenation-Induced Apoptosis via the AKT/Nox2 Pathway. Oxid Med Cell Longev. 2019;2019:2717986.

12. Rurali E, Pilato CA, Perrucci GL, Scopece A, Stadiotti I, Moschetta D, Casella M, et al. Cyclophilin A in arrhythmogenic cardiomyopathy cardiac remodeling. Int $\mathbf{J}$ Mol Sci. 2019;20(10).

13. Hoffmann H, Schiene-Fischer C. Functional aspects of extracellular cyclophilins. Biol Chem. 2014;395(78):721-735.

14. Satoh K, Fukumoto Y, Sugimura K, Miura Y, Aoki T, Nochioka K, Tatebe S, et al. Plasma cyclophilin A is a novel biomarker for coronary artery disease. Circ J. 2013;77(2):447-455.

15. Liu X, Du H, Chai Q, Jia Q, Liu L, Zhao M, Li J, et al. Blocking mitochondrial cyclophilin D ameliorates TSH-impaired defensive barrier of artery. Redox Biol. 
2018;15:418-434.

16. Porter GA, Jr., Beutner G. Cyclophilin D, somehow a master regulator of mitochondrial function. Biomolecules. 2018;8(4).

17. Alfonso A, Bayon J, Gegunde S, Alonso E, Alvarino R, Santas-Alvarez M, Testa-Fernandez A, et al. High Serum Cyclophilin C levels as a risk factor marker for Coronary Artery Disease. Sci Rep. 2019;9(1):10576.

18. Roffi M, Patrono C, Collet JP, Mueller C, Valgimigli M, Andreotti F, Bax JJ, et al. 2015 ESC Guidelines for the management of acute coronary syndromes in patients presenting without persistent ST-segment elevation: task force for the management of acute coronary syndromes in patients presenting without persistent ST-segment elevation of the European Society of Cardiology (ESC). Eur Heart J. 2016;37(3):267-315.

19. Ibanez B, James S, Agewall S, Antunes MJ, BucciarelliDucci C, Bueno H, Caforio ALP, et al. 2017 ESC Guidelines for the management of acute myocardial infarction in patients presenting with ST-segment elevation: The Task Force for the management of acute myocardial infarction in patients presenting with ST-segment elevation of the European Society of Cardiology (ESC). Eur Heart J. 2018;39(2):119-177.

20. Task Force Members, Montalescot G, Sechtem U, Achenbach S, Andreotti F, Arden C, Budaj A, et al. 2013 ESC guidelines on the management of stable coronary artery disease: the Task Force on the management of stable coronary artery disease of the European Society of Cardiology. Eur Heart J. 2013;34(38):2949-3003.
21. Seizer P, Fuchs C, Ungern-Sternberg SN, Heinzmann D, Langer H, Gawaz M, May AE, et al. Platelet-bound cyclophilin A in patients with stable coronary artery disease and acute myocardial infarction. Platelets. 2016;27(2):155158.

22. Alam MR, Baetz D, Ovize M. Cyclophilin D and myocardial ischemia-reperfusion injury: a fresh perspective. J Mol Cell Cardiol. 2015;78:80-89.

23. Ridker PM, Everett BM, Thuren T, MacFadyen JG, Chang WH, Ballantyne C, Fonseca F, et al. Antiinflammatory therapy with canakinumab for atherosclerotic disease. N Engl J Med. 2017;377(12):1119-1131.

24. Schofer N, Ludwig S, Rubsamen N, Schnabel R, Lackner KJ, Ruprecht HJ, Bickel C, et al. Prognostic impact of Interleukin-1 receptor antagonist in patients with documented coronary artery disease. Int J Cardiol. 2018;257:24-29.

25. Danesh J, Kaptoge S, Mann AG, Sarwar N, Wood A, Angleman SB, Wensley F, et al. Long-term interleukin-6 levels and subsequent risk of coronary heart disease: two new prospective studies and a systematic review. PLoS Med. 2008;5(4):e78.

26. Walter J, Tanglay Y, du Fay de Lavallaz J, Strebel I, Boeddinghaus J, Twerenbold R, Doerflinger S, et al. Clinical utility of circulating interleukin- 6 concentrations in the detection of functionally relevant coronary artery disease. Int J Cardiol. 2019;275:20-25.

27. Bartekova M, Radosinska J, Jelemensky M, Dhalla NS. Role of cytokines and inflammation in heart function during health and disease. Heart Fail Rev. 2018;23(5):733758 . 